The present way of treating these cases was also the old way used by the majority of the the defects in these tissues also may be considprofession. The very first thing is opium in erable.

some form to relieve the pain, as they will tell It would thus seem natural to select a method you in most cases without looking for a hernia, of operating by which no tissue should be lost. or any other cause for the trouble. Pain is the In all the older methods the so called "freshen. only symptom complained of, so most ment are content when the patient is relieved of that.

I have been called to a patient suffering from strangulated hernia, with all the symptoms of peritonitis, and the medical attendant not aware he had anything more than a case of idiopathic peritonitis to deal with. I will venture to say, that if all the medical men in this city could be asked what the first thing they would do for a case of threatened peritonitis, they would say relieve him or her of pain by opium. It is the almost universal rule, notwithstanding the stress laid on the great danger to the patient in such treatment.

There is still another and most important reason for early treatment in these cases. We all know that one of the favorite and accepted theories of the introduction of tubercle bacillus is through the stomach in its deranged and diseased condition from any cause, and in this way into the general circulation to be deposited in that portion least able to resist its ravages. In these cases where we have a subacute inflammatory condition with large deposits of inflammatory matter of low vitality, where the circulation is in a most unsatisfactory condition, where the thermometer indicates a temperature in which these little bodies grow and flourish to a most alarming extent, tissues of low vitality are fastened together. What better culture ground or condition could even a pathologist wish for in which to feed and grow his medical menagerie. Be this true or not, the fact still stands forth that many of these chronic cases die of tuberculosis.

\title{
A NEW OPERATION FOR HARELIP.
}

Read in the Section of Surgery and Anatomy, at the Forty-second $A n$ nual Meeting of the American Medical Association, held at Washington, $D$. C., May 5,189 .

BY CHRISTIAN FENGER, M.D., OF CHICAGo, ILL.

In all forms of harelip (labium leporinum, whether single or bilateral, whether partial or total, whether combined with cleft inter-maxillary bone or not), we find not an excess, but rather a defect of labial tissue, mucous membrane, prolabium and skin. The two portions of the upper lip, when brought together, are too small to form a lip of normal shape; it would require the interposition of a triangular piece of lip with apex towards the nose and base toward the border of the lip, to obtain at once a lip of normal shape. The tissue defect is greatest in the skin, less manifest in the mucous membrane and prolabium; ing' of the surfaces to be united requires the removal of at least a part of the prolabium, in some more, in others less, at the best, as in the method of Nélaton for small harelip, a displacement downward, to form a projection which must either disappear by retraction and atroply, or be removed, if present in excess, by a secondary operation.

About five years ago I was led to consider this question in a case of double harelip with rather defective lateral portions, in which I operated after the old method and suppuration with consequent non-union took place. At the next attempt at union, the increased defect of tissue made union still more difficult than at the first operation. I therefore concluded to operate in my next case without the removal of any tissue, so as to have at least not lost any thing in this respect if suppuration and non-union should make one or more later operations necessary. When I commenced to operate in this way I found it necessary to apply a separate row of sutures to the prolabium before bringing the edges of the skin together, thus closing the wound toward the mouth, and I soon found this to be a protection against infection.

I have since that time never had any reason to abandon this method for any of the older methods.

\section{OPERATION FOR UNIIATERAL HARELIP.}

I. Incision.-The incision is macle at the border of the skin and prolabium, four to six millimeters or two to three lines deep, care being taken to guard against opening through the mucous membrane into the mouth.

It is necessary to draw the lip down in order to procure sufficient tension for the knife to divide the tissues; this traction causes anæmia of the border of the lip, making the line between the white skin and red prolabium indistinct. It is necessary to be careful to have the incision precisely in this line, because a small margin of prolabial tissue left with skin will, as I have seen in one case, leave a red brim along the line of union, necessitating a secondary operation for cosmetic reasons. (Figure I.)

The length of the incision downward and outward depends upon the shape of the two portions of the separated lip. It is unnecessary at the beginning of the operation to estimate the length correctly, as the incision can be prolonged later on, after some of the sutures have been applied and tied, according to the requirements of the shape of the lip when united.

2. Sutures of the Prolabium.-Interrupted 
sutures of fine silk are applied or inserted from the mucous surface so as to place the knot of the suture in the mouth, the first suture close to the top of the triangle as shown in Figure 2. The ends of the sutures are left long and held together with an artery forceps which is left hanging down. The succeeding sutures are applied in a similar manner at a distance of from three to five millimeters from each other in a number corresponding to the extent of the line of prolabium to be minited. These prolabial sutures should include the mucous membrane and sub mucous tisste only, or as little tissue as will suffice to keep the prolabial edges together. There must be no tension whatever at the line of union; if tension exists from shortness of the labial flaps, it must be overcome by the tension sutures, which will be described later.
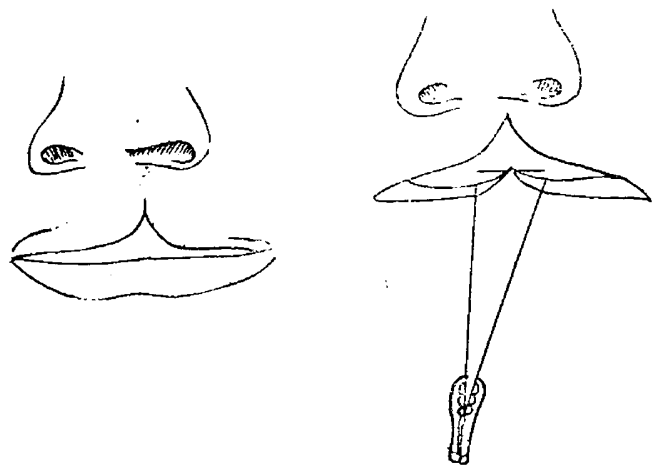

Figure $\mathrm{I}$.

Figure 2

When apparently a sufficient number of these sutures have been applied, they are tied from above downward, or from the nose to the prolabium, the upper or nasal suture first, the forceps being held up toward the forehead. The united line must be long enough to give sufficient length to the lip, and permit the lowest point of union to be a little lower down (toward the lower lip), than the sides of the lip and the corners of the mouth. The wound to be united is now closed against the mouth -is made a sub cutaneous wound.

Sutures of the Skin.-I. Tension Sutures. According to the size of the defect, one or two tension sutures become necessary. I have entirely discarded the figure-of eight suture with harelip pins, and also the button and shot sutures, and always employ an interrupted suture, using silk a little heavier than that tosed for the coaptation sutures of the prolabium and skin. In ordinary cases of single harelip one tension suture is sufficient. Tinis suture enters the skin at a distance of one quarter to half an inch from the cutaneous margin of the wound, and is passed deeply down into the tissue of the lip. Care is taken not to have it pass through to the mouth, but to have it take in about two-thirds of the tissue of the lip as shown in Figure 4 a. This tension suture should be placed at or near a point where the margins of the wound are the greatest distance from each other-that is, near to the prolabium of the undivided portion of the lip. The tension suture is left with long ends held by artery forceps, and should not be tied until all the cutaneous coaptation sutures have been inserted.

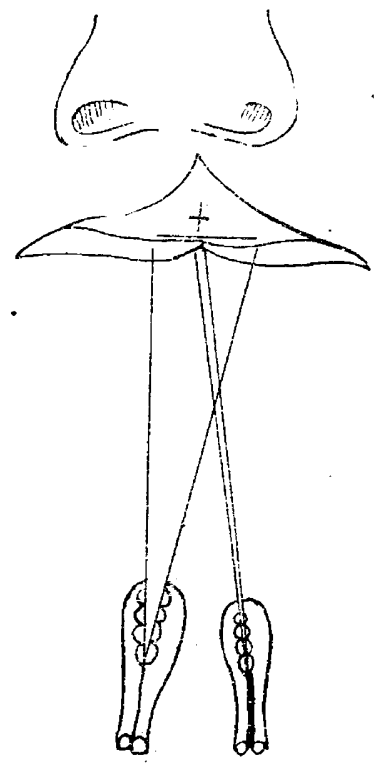

Figure 3 .

2. Coaptation Sutures. These sutures (Figure 4, b.b.b.), for which common silk twist (which may be bought in a dry goods store, and disinfected in the usual manner by boiling it from fifteen to thirty minutes in a 5 per cent. solution of carbolic acid) is used, are applied from above downward, or from the nasal angle of the wound to the prolabium, at a distance of three to five millimeters. Three to five sutures are usually required. The lowest suture (Figure $4, \mathrm{c}$ ), is passed through the ununited borders of the prolabium.

The wound is now carefully cleansed by small aseptic sponges, and is finally touched with a sponge wrung out from $21 / 2$ per cent. of carbolic acid, or a $\mathrm{I}$ to $2, \mathrm{coO}$ sublimate solution.

If any hæmorrhage is present it can be stopped by continued pressure with an aseptic sponge for a few minutes; if the hæmorrhage is slight it will cease upon tying the sutures.

The closure of the sutures should begin with the tension suture, so as to prevent the coaptation sutures from tearing through, as they would be liable to do if tied before all tension was done away with. 
If in drawing the sutures together it is found that the middle of the lip does not come down low enough, or that there is still an indentation in the prolabium at the line of incision, it is advisable to prolong the cutaneo-prolabial incision a little on one or both sides, as the shape of the lip may require, and then apply an additional cutaneous coaptation suture.

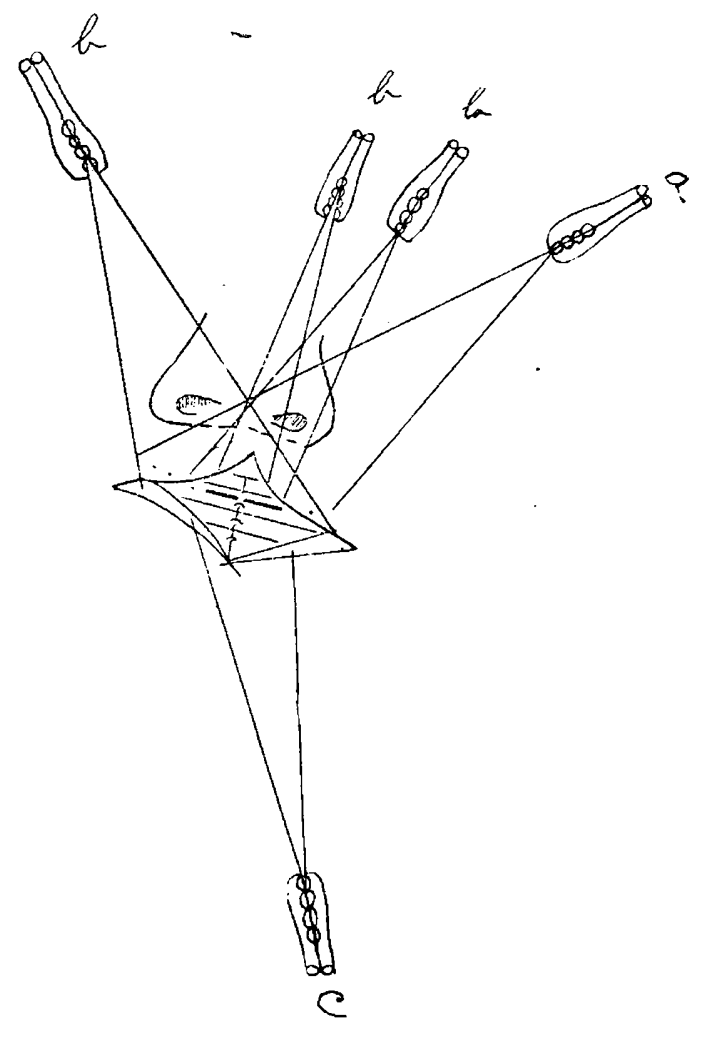

Figure 4.

When the cutaneous sutures are tied, the united wound should present the appearance shown in Figure 5.

4. Dressing of the Wound.-After careful cleansing of the line of the wound with saturated solution of boracic acid, a layer of finely powdered boracic acid is dusted on, and a cotton-collodion dressing applied. When I began to use this method I plugged the nostril with borated cotton, but on account of its inconvenience to the patient, have now discontinued this practice. I do not use iodoform-cotton or iodoform-collodion dressing for fear of iodoform poisoning; I prefer to have the nurse or mother dust finely powdered boracic acid into the nostril every three or four hours during the day.

A single or double strip of rubber adhesive plaster, extending from ear to ear, is applied over the collodion dressing in such a manner as to draw the two cheeks somewhat together, for the purpose of immobilizing the cheeks and lips when the child cries or nurses.

The dressing may be changed once a day, or every two or three days if it remains dry. If, however, the dressing becomes soaked by nasal secretion, or milk when the child is nursed, it should be changed according to the necessity of the individual case.

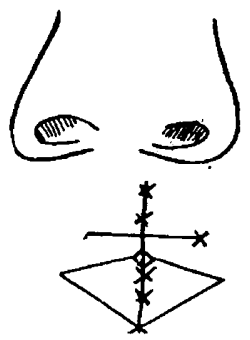

Figure 5

5. Removal of Sutures. -The cutaneous coaptation sutures are removed after a week; the tension suture is allowed to remain two to four days longer if it has not been loosened by pressure atrophy, or pressure necrosis of its canal. After removal of the sutures, the borated cottoncollodion dressing and adhesive straps are reapplied to be continued until the suture canals have entirely healed, which takes place toward the end of the second or third week.

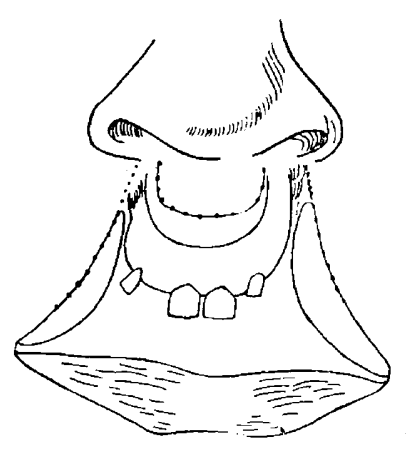

Figure 6.

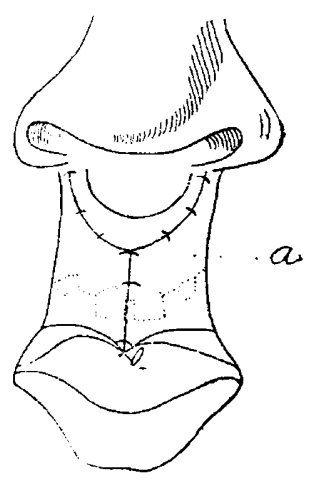

Figure 7 .
At the junction of the two lateral corners of the wound there often remains after the suturing a small quadrangular ununited space one to two millimeters in diameter, where it may have been impossible to approximate the edges of the wound. (Figure 5 a.). A very fine suture may belp to close this little defect. If, as is often the case, this little suture cuts through, the small defect heals by aseptic granulation, requiring only very little more time than the primary union of the remainder of the wound. It leaves at most a small cicatrix in the line of union at the border of the skin and prolabium, and does not result in permanent disfigurement. 
The prolabial sutures will sometimes cut through and peel off if the process of healing goes on undisturbed, or some of the sutures may remain and be removed after the end of the second or third week, at a time when union is strong enough to tolerate the manipulations necessary for their removal.

\section{OPERATION FOR DOUBLE AND COMPLICATED HARELIP.}

The operation for double and complicated harelip (as represented in Figures 6 to 9), is performed on the same general principles, namely:

I. Incision.- The incision along the cutaneoprolabial border should be of sufficient length to cover the space of defect. A second incision should be made along the cutaneo-prolabial border of the median peninsula of labial tissue below nasal septum, if this peninsula is so situated as to be available for use in the formation of the lip. (See Figure 6).

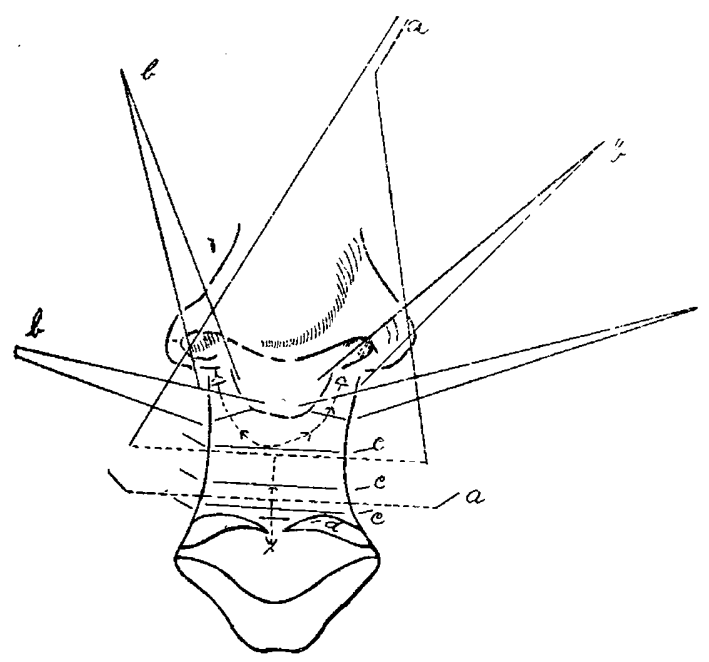

Figure 8.

2. Suture of the Prolabium.-The prolabial sutures are applied as just described for the unilateral harelip, to be tied on the oral surface of the wound. The sutures should be first inserted on both sides of the nasal peninsula until this is wholly covered, and until both lateral halves of the prolabium cone together at the median point of its lower border. (Figure 7, a.). When this point is reached these two rows of sutures are tied.

The lateral prolabial borders are now united in the median line as low down or as far out as required to give to the lip the necessary length, as described in the operation for single harelip. These labial sutures are tied in the mouth usually without any tension, because the prolabium when loosened from the skin and retroverted, furnishes a flap of sufficient size and elasticity to permit of ready approximation.

It is important, as before stated, that the linear incision should not penetrate into the mouth. When, as is often the case when the cleft extends through the nostril or nasal cavity, there is a lack of prolabium near to and in the nostril, and the prolabial sutures cannot be applied high up, it is usually possible, by careful dissection and separation of the mucous membrane at the oral fornix of the lateral half of the upper lip, to displace the mucous membrane to some extent laterally, so that the uppermost suture may be applied reasonably high up toward the nostril.

When the prolabial sutures have been tied the wound presents the appearance represented in Figure 7 .

3. Sutures of the Skin.-Tension and coaptation sutures are now applied as follows: (See Fgure 8).

I. Tension Sutures.-For these sutures somewhat heavier silk should be employed. The upper suture should be inserted at the lower border of the nasal peninsula, the lower one near

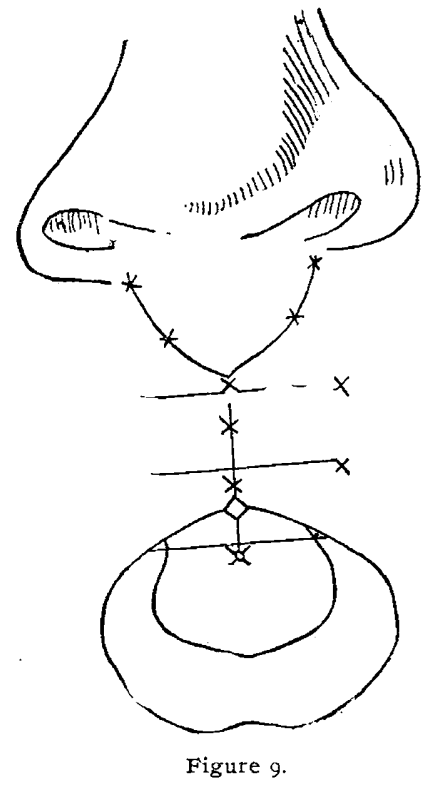

the lower border of the lip. They should be passed in and out at a distance of a quarter to half an inch of the border of the skin, and should include one-half or a little more than one-half of the thickness of the lip. (Figure 8 , a.) The ends should be held by artery forceps, and should not be tied until all the skin sutures have been applied.

2. Coaptation Sutures. - The coaptation sutures (Figure 8, b. c.), are now inserted from above downward. The first two or three sutures (Figure 8. b.), are inserted on both sides between the lateral skin flap and the skin of the peninsula, until this is united to the side flap throughout its entire circumference. The lateral flaps, uniting in the median line just below the nasal peninsula, forms the upper portion of a Y- 
shaped line of union. Then the lower portion of the wound is united by two or three sutures (Figure 8, c.), and a lower fourth suture (Figure 8 , d.) is inserted through the two halves of the prolabium.

After thorough cleansing and disinfection of the wound, the sutures should be tied : first, the tension, and then the coaptation sutures (Figure 7).

4. Dressing of the Wound.-The wound should be dressed with borated cotton-collodion, or in older patients, with iodoform collodion. The nostrils, especially in older patients, should be loosely packed with borated or iodoform cotton. Over this two straps of adhesive plaster should be applied. In older patients a strip of iodoform gauze should be laid in the mouth along the line of union of the prolabium. In new-born children the nostrils need not be packed with cotton, and no iodoform gauze should be used; but these localities should be frequently dusted with finely powdered boracic acid. The dressing should be changed according to the amount of discharge, never more than once daily.

5. Removal of Sutures. - The coaptation sutures of the skin may be removed at the end of the first week; the tension sutures, according to the amount of tension remaining, usually late in the second week; finally, what may remain of the prolabial sutures should be removed still later, when the union is so solid that the upper lip is able to tolerate manipulation.

It is immaterial how narrow the new upper lip may appear to be immediately after the operation. König remarks (in speaking on plastic operations on the lips-keiloplastic operations following the extirpation of labial carcinomas), that the shape of the mouth, however deformed it may appear, on account of unequal size of the lower and upper lip in the first weeks after the operation, that in the course of some months the shape of the oral orifice and lips becomes more normal, surprisingly so when a sufficient time, from three to six months, has elapsed.

The all-important feature of the operation is to make the upper lip long enough in a sagittal direction, and to bave exact union of the lower portion of the prolabium.

Apparatus to draw the cheeks together for the sake of immobilization or to diminish tension, I have, up to this time, found superfluous.

\section{CONCLUSIONS.}

The two main points in this harelip operation are the incision and the prolabial sutures.

I. The incision, a linear incision, is similar to that made in the operation for laceration of the perineum as proposed by Lawson Tait, and by him termed "flap-splitting." As stated above, this possesses the advantage of not sacrificing any tissue of the parts already more or less de- fective; on the other hand, repeated denudations made necessary by unsuccessful attempts at union would tend to make the lateral portions of the lip smaller and smaller. Furthermore, the linear incision, by loosening the everted prolabium (which is readily converted into mucous membrane by the simple change of position into the oral cavity), furnishes so much tissue for the posterior surface of the lip that union of the prolabial borders is easy, even before any tension sutures have been applied.

2. The prolabial sutures should be accurately applied. Fine curved needles should be used, the sutures made of fine silk. Interrupted sutures should be employed, in sufficient number and at sufficient intervals to furnish exact union. These sutures close the wound against the mouth and make the wound, so to speak, cutaneous in. stead of visceral, and thereby prevent infection from the ever-present multitude of microbes in the mouth. I believe that this suture will prove to be a potent factor in securing uniform results by preventing suppuration and non-anion of the wound.

3. The cosmetic results have been all I could desire. If a slight indentation at the line of union of the prolabium has existed immediately after the operation, it has generally disappeared after a few months, provided the lip bas been made long enough at the time of the operation. 4. I think that this method is applicable in all cases, and should take the place of all the numerous older methods of operating.

\section{SPRAINS OF THE ANKLE.}

Read in the Section of Surgery and Anatomy, at the Forty-second Annual Meeting of the American Medical Association, held at Washington, D. C., May, I891.

BY W. R. TOWNSEND, A.M., M.D., OF NEW YORK.

ASSISTANT SURGEON, HOSPITAL FOR RUPTURED AND CRIPPIED; ORTHOPADIC SURGEON, NEW YORK INFANT ASYLUM; LECTLLER ON ORTHOPADIC SUKGERY, NEW YORK POLYCLINIC.

There is a popular impression among many of the laity "that a sprain is worse than a fracture," yet, to judge from the amount of space devoted to the subject in our text-books on surgery, it is evident that their authors do not agree with the statement. We must, however, admit that some grounds exist for the belief, and that, while we seldom hear of an ununited fracture, or of a fracture causing trouble years after the original injury, we are constantly meeting with cases of chronic sprain of the ankle, of flat foot, of various forms of bone and joint disease which we can clearly trace to a sprain. Several reasons exist for this state of affairs, and in many instances, the reason may be clearly shown to be the faulty or neglected treatment of the original injury.

Mr. Mansell Moullin, of London, in his most thorough and interesting monograph on "Sprains; 